\title{
miR-145-5p inhibits the proliferation and migration of bladder cancer cells by targeting TAGLN2
}

\author{
HAIJIAN ZHANG ${ }^{1 *}$, MEIJUAN JIANG ${ }^{2 *}$, QINGJUN LIU ${ }^{1}$, \\ ZHIXING HAN $^{1}$, YUQIAN ZHAO ${ }^{1}$ and SHIQI JI ${ }^{1}$ \\ Departments of ${ }^{1}$ Urology and ${ }^{2}$ Neurology, Beijing Ditan Hospital, \\ Capital Medical University, Beijing 100015, P.R. China
}

Received March 13, 2018; Accepted August 28, 2018

DOI: $10.3892 / \mathrm{ol} .2018 .9436$

\begin{abstract}
MicroRNA-145-5p (miR-145-5p) is found to be involved in tumor development and progression. However, there are few studies on the effects of miR-145-5p on bladder cancer (BC). The role of miR-145-5p in BC was predicted by analysis of cell proliferation and migration in this study. The miR-145-5p and transgelin-2 (TAGLN2) expressions were evaluated via reverse transcription-quantitative PCR (RT-qPCR) or western blot analysis. The MTT and Transwell assay assessed cell proliferation and migration. TAGLN2 targeted to miR-145-5p was determined using luciferase assays. The results showed that the miR-145-5p downregulation was found in BC. miR-145-5p overexpression inhibited cell proliferation and migration in BC. Moreover, miR-145-5p directly targeted TAGLN2, and TAGLN2 expression was increased in BC. In addition, the high expression of TAGLN2 promoted cell proliferation and migration in BC. miR-145-5p appeared to regulate TAGLN2 in $\mathrm{BC}$, and it also inhibited the cell proliferation and migration. The novel miR-145-5p/TAGLN2 axis may provide new therapeutic implications for $\mathrm{BC}$.
\end{abstract}

\section{Introduction}

As a typical malignant urogenital tract cancer, bladder cancer (BC) is the sixth most prevalent cancer (1) and the second most common cause of death for cancer in the urinary system for men (2). About 330,000 new BC cases occur around the world resulting in 130,000 deaths annually (3). The main pathogenic factors of bladder are environmental and genetic factors (4). Although surgical operation, radiotherapy and

Correspondence to: Dr Shiqi Ji, Department of Urology, Beijing Ditan Hospital, Capital Medical University, 8 Jingshun East Street, Chaoyang, Beijing 100015, P.R. China

E-mail: sk72sb@163.com

${ }^{*}$ Contributed equally.

Key words: miR-145-5p, transgelin-2, proliferation, migration, bladder cancer chemotherapy have made great improvement in treatment, $\mathrm{BC}$ is still a common disease with a high mortality rate (5). Moreover, there are many limitations for the established BC biomarkers to diagnose the various cancers (6). Thus efficient gene therapies should be developed to use for early diagnosis of cancer.

MicroRNAs (miRNAs) act as tumor suppressors or oncogenes by regulating gene expression at the post-transcriptional levels to get involved in tumor formation (7). Especially, dysregulation of miRNAs in BC was closely related with bladder tumorigenesis (8). Therefore, miRNAs could be used to diagnose cancer. There are many studies on the role of miRNAs which influenced cell physiological activity in BC, such as cell migration, invasion, proliferation and survival. For example, tumor suppressors including miR-106a, miR-223, and miR-613 accommodated BC by regulating the MAPKs, NCOA1 and SphK1, respectively (9-11). In addition, tumor oncogenes containing miR-130, miR-200c, and miR-556 regulate $\mathrm{BC}$ by regulating the PTEN, RECK and DAB2IP, respectively (12-14). However, research on the function of miR-145-5p is still rare in the pathogenesis of BC.

Transgelin-2 (TAGLN2) belonging to the ABP family was firstly discovered in 1998 (15). Recently, the dysregulated expression of TAGLN2 was identified in various cancers. TAGLN2 function as an oncogene to promote cancer cell proliferation, invasion and migration (16-18). In addition, Yoshino et al indicated that TAGLN2 along with miR-1/133a affected cell proliferation, apoptosis, invasion and migration in BC (19). Nonetheless, there are no reports on TAGLN2/miR-145-5p in BC.

This study proposed the hypothesis that miR-145-5p suppressed tumor formation by regulating TAGLN2. In order to verify the hypothesis, miR-145-5p and TAGLN2 expressions were detected in BC. Besides, we also examined cell proliferation and migration to further expound the roles of miR-145-5p and TAGLN2 in BC.

\section{Materials and methods}

Clinical tissues. Clinical tissues were collected from 22 patients with $\mathrm{BC}$ who were undergoing transurethral resection or cystectomy at Beijing Ditan Hospital, Capital Medical University (Beijing, China). The study was approved by the 
A

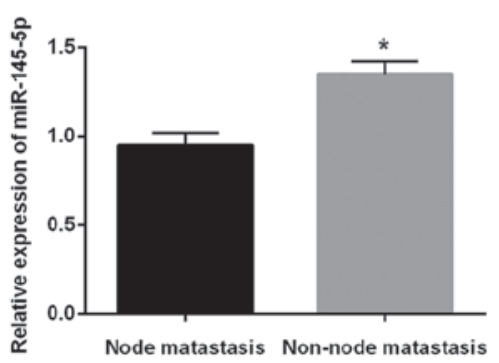

B

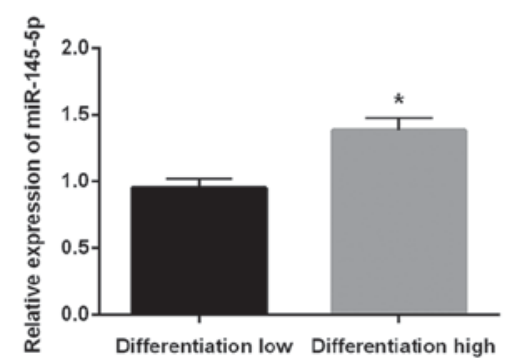

C

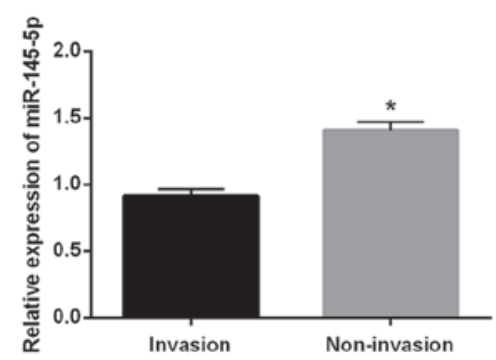

Figure 1. The relationship between the miR-145-5p expression and the clinicopathological features was analyzed in BC. (A) The correlation between miR-145-5p and lymph node metastasis. (B) The association between miR-145-5p and vessel invasion. (C) The association between miR-145-5p and differentiation. ${ }^{*} \mathrm{P}<0.05$; $\mathrm{BC}$, bladder cancer.

Ethics Committee of Beijing Ditan Hospital, Capital Medical University. Signed written informed consents were obtained from the patients or guardians. BC adjacent tissues were obtained from areas about $2 \mathrm{~cm}$ away from tumor lesions.

Cell culture and transfection. The human BC cell lines T24 and 5637, and the immortalized urothelial cell line SV-HUC-1 were applied in this study. All the cell lines were obtained from the Shanghai Institute of Cell Biology, Chinese Academy of Sciences (Shanghai, China). The cells were grown in RPMI-1640 medium supplemented by $10 \%$ fetal bovine serum (FBS). The cells were incubated at $37^{\circ} \mathrm{C}$, with $5 \% \mathrm{CO}_{2}$ atmosphere.

The miR-145-5p mimic and inhibitor, TAGLN2 siRNA were purchased from RiBoBio Co., Ltd. (Guangzhou, China) and then they were transferred into T24 or 5637 cells with Lipofectamine ${ }^{\circledR} 2000$ (Invitrogen; Thermo Fisher Scientific, Inc., Carlsbad, CA, USA) according to manufacturer's instructions.

RNA extraction and reverse transcription-quantitative PCR $(R T-q P C R)$. TRIzol reagent (Invitrogen; Thermo Fisher Scientific, Inc.) was applied for extracting total RNA containing miRNA to quantitate the miR-145-5p expression in BC tissues and cell lines. RNA was reverse transcribed using the RevertAid First Strand cDNA kit (Thermo Fisher Scientifc, Inc.) according to the manufacture's introduction.The reactions were incubated at $25^{\circ} \mathrm{C}$ for $5 \mathrm{~min}, 42^{\circ} \mathrm{C}$ for $60 \mathrm{~min}$ and $70^{\circ} \mathrm{C}$ for $5 \mathrm{~min}$. The $\mathrm{miR}-145-5 \mathrm{p}$ reverse transcription primer is $5^{\prime}-\mathrm{AGT}$ CCAGTTTTCCCAGGAATCCCT-3'. RT-qPCR was performed through the SYBR Premix Ex Taq (Takara Bio, Inc., Otsu, Japan) on an ABI PRISM 7500 Real-time PCR system (Applied Biosystems, Thermo Fisher Scientific, Inc.). The reactions were incubated at $94^{\circ} \mathrm{C}$ for $10 \mathrm{~min}$, followed by 45 cycles at $95^{\circ} \mathrm{C}$ for $15 \mathrm{sec}$ and $60^{\circ} \mathrm{C}$ for $30 \mathrm{sec}$. The primers were designed as follows: miR-145-F 5'-CCTTGTCCTCAC GGTCCAGT-3', and R 5'-AACCATGACCTCAAGAACAGT ATTT-3'; TAGLN2-F 5'-CTACCTGAAGCCGGTG TCC-3', and R 5'-ATCCCCAGAGAAGAGCCCAT-3'; U6-F 5'- GCT TCGGCAGCACATATACTAAAAT -3', and R 5'- CGCTTC ACGAATTTGCGTGTCAT -3'; GAPDH-F, 5'-GAGTCA ACGGATTTGGTCGT-3' and R, 5'-TTGATTTTGGAGGGA TCTCG-3'. U6 and GAPDH were used as control of miR-145 and TAGLN2. The miR-145-5p and TAGLN2 levels were analyzed using the $2^{-\Delta \Delta \mathrm{Cq}}$ method (20).
Cell proliferation and migration assays. The cell proliferation and migration were performed using MTT and Transwell assays to investigate the role of the miR-145-5p and TAGLN2 using T24 or 5637 cells. The experimental procedures were performed according to a previous study (18).

Dual luciferase report assays. The wt 3'-UTR of TAGLN2 or mut 3'-UTR of TAGLN2 were inserted into the pGL3 promoter vector (GenScript, Nanjing, China) for luciferase reporter experiments. Then, the vector and miR-145-5p mimic were transfected into 5637 cells. Cells were cultured in a 24-well plate. About $48 \mathrm{~h}$ after transfection, the dual luciferase reporter assay system (Promega Corporation, Madison, WI, USA) was applied to perform luciferase assays.

Western blot analysis. The protein samples were obtained using RIPA buffer. Proteins were separated through SDS-PAGE and then incubated with 5\% non-fat milk blocked membranes at room temperature. Next we incubated the membranes overnight at $4^{\circ} \mathrm{C}$ with rabbit polyclonal TAGLN2 antibody (cat. no. ab233478; 1:1,000), rabbit polyclonal GAPDH antibody (cat. no. ab9485; 1:1,000) and subsequently incubated with matched goat polyclonal secondary antibody to rabbit IgG - H\&L (cat. no. ab150077; 1:1,000) all from Abcam (Cambridge, MA, USA). The protein expression levels were measured by a gel imaging system (JS-780; Pei Qing Technology Co., Ltd., Shanghai, China).

Statistical analysis. The experimental data are presented as the mean \pm SD. Enumeration data were analyzed using Student's t-test and Chi-square test. Statistical analysis was analyzed with GraphPad Prism 6.0 (GraphPad Software, Inc., La Jolla, CA, USA) and SPSS 17.0 (SPSS, Inc., Chicago, IL, USA). $\mathrm{P}<0.05$ was considered to indicate a statistically significant difference.

\section{Results}

The relationship between $m i R-145-5 p$ expression and the clinicopathological features was analyzed in BC. In Fig. 1, the relationships between clinicopathological characteristics and the expression of miR-145-5p are summarized. The miR-145-5p expression was closely related with lymph node metastasis (Fig. 1A), differentiation (Fig. 1B) and vascular invasion (Fig. $1 \mathrm{C} ; \mathrm{P}<0.01$ ). 
A

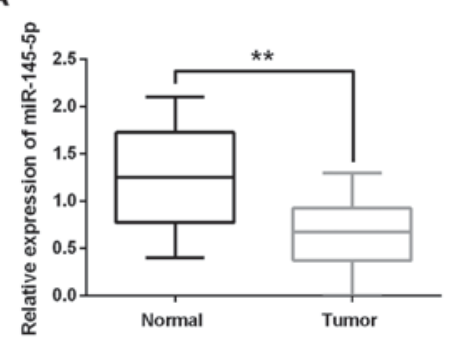

C

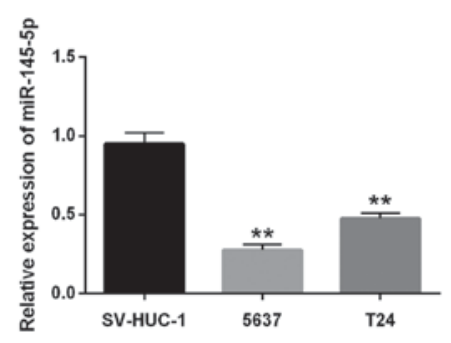

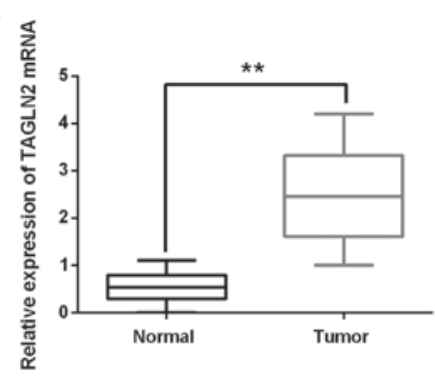

D

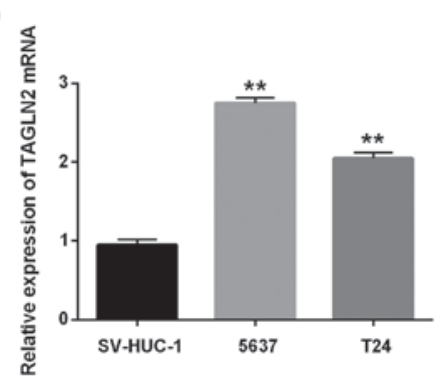

Figure 2. The expression of miR-145-5p and TAGLN2 in BC. (A and B) The miR-145-5p and TAGLN2 expression in tumor tissues and corresponding non-tumor tissues. (C and D) The miR-145-5p and TAGLN2 expressions in T24, 5637 and SV-HUC-1 cells (control). ${ }^{* *} \mathrm{P}<0.01 ; \mathrm{BC}$, bladder cancer.

A

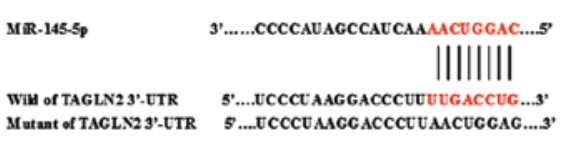

C

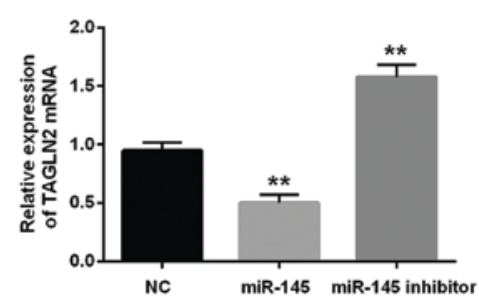

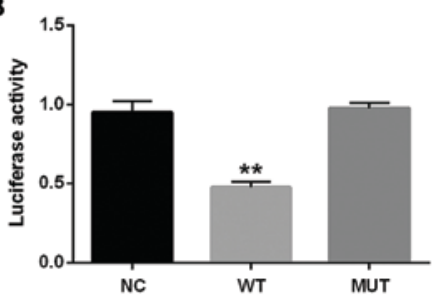

D

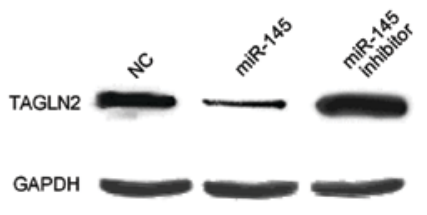

Figure 3. miR-145-5p directly targets TAGLN2 in BC cells. (A) The binding sites of miR-145-5p and TAGLN2. (B) Luciferase reporter assay. (C and D) The mRNA and protein expression of TAGLN2 were analyzed in cells containing miR-145-5p mimic or inhibitor. ${ }^{* *} \mathrm{P}<0.01$; $\mathrm{BC}$, bladder cancer.

miR-145-5p and TAGLN2 expression in BC tissues and cells. The miR-145-5p and TAGLN2 expression in BC tissues was detected using RT-qPCR. The miR-145-5p expression was lower than that of normal tissues in $\mathrm{BC}$ tissues but higher than expression of TAGLN2 (Fig. 2A and B; $\mathrm{P}<0.01$ ). Furthermore, the miR-145-5p and TAGLN2 expression in BC cell lines was detected simultaneously. The BC cell lines (5637 and T24) had low expression for miR-145-5p but high expression for TAGLN2 mRNA (Fig. 2C and D; $\mathrm{P}<0.01$ ). Because the expression levels of 5637 were considered to have more difference than T24, 5637 cell line was selected in the following experiments.

miR-145-5p directly targets TAGLN2 in BC cells. TargetScan database predicted that TAGLN2 3'UTR has a binding site for
miR-145-5p (Fig. 3A). To verify the above result, we conducted luciferase reporter assay in the 5637 cells. As predicted, luciferase activity in the cells transfected with miR-145-5p mimic and wild-type TAGLN2 was distinctly deceased compared to the control group $(\mathrm{P}<0.01)$. Additionally, little change was found in cells containing mutated TAGLN2 and miR-145-5p mimic (Fig. 3B; P>0.05). Furthermore, miR-145-5p overexpression downregulated TAGLN2 mRNA and protein expression significantly (Fig. 3C and D).

miR-145-5p suppresses the cell proliferation and migration in $B C$. The function of miR-145-5p for regulating the cell proliferation and migration in $\mathrm{BC}$ were investigated in the present study. The high transfection efficiency was detected in cells containing miR-145-5p mimics or inhibitor (Fig. 4A). 
A

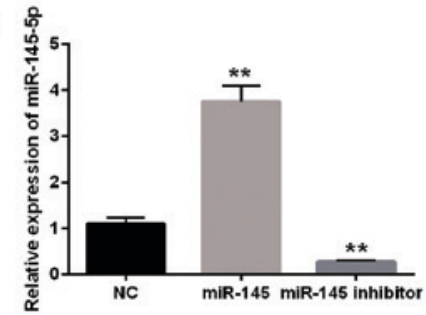

C

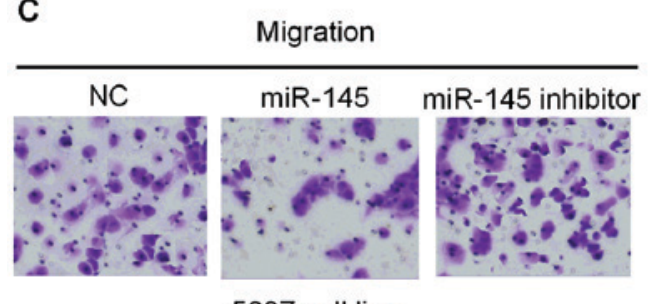

B

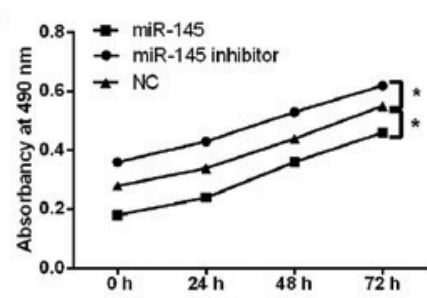

D

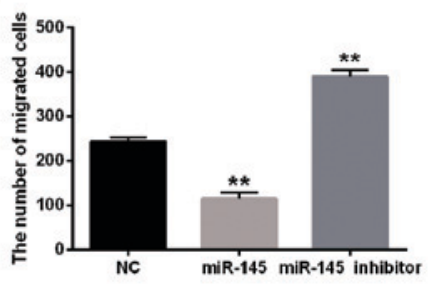

Figure 4. miR-145-5p suppresses BC cell proliferation and migration. (A) miR-145-5p expression was examined in cells contained miR-145-5p mimic or inhibitor via RT-qPCR. (B) The cell proliferation was measured in cells contained miR-145-5p mimic or inhibitor via MTT. (C and D) The cell migration was measured in cells contained miR-145-5p mimic or inhibitor via Transwell analysis. ${ }^{*} \mathrm{P}<0.05 ;{ }^{* * *} \mathrm{P}<0.01 ; \mathrm{BC}$, bladder cancer.

A

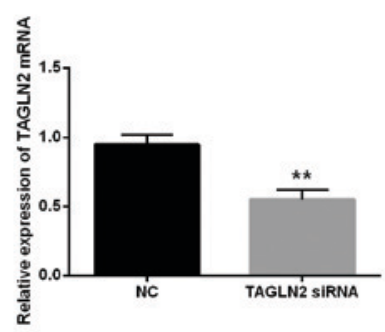

C

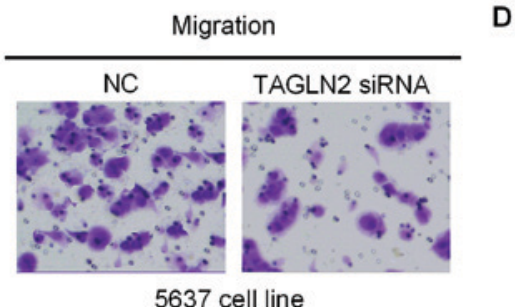

B

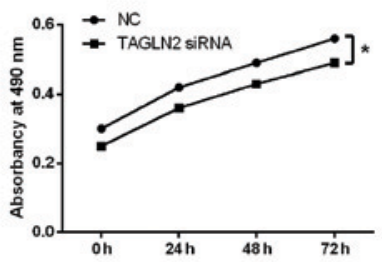

D

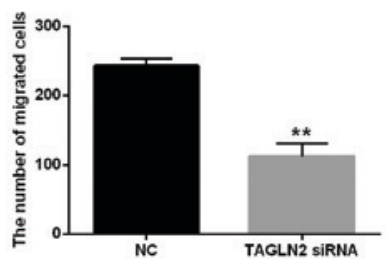

Figure 5. TAGLN2 promotes BC cell proliferation and migration. (A) Expression levels of TAGLN2 were examined in cells contained TAGLN2 siRNA via RT-qPCR. (B) The cell proliferation was measured in cells containing TAGLN2 siRNA via MTT. (C) The cell migration was measured in cells containing TAGLN2 siRNA via Transwell analysis. (D) The number of migrated cells after treatment with TAGLN2 siRNA. "P<0.05; ${ }^{* *} \mathrm{P}<0.01$.

Moreover, the MTT results demonstrated that cell proliferation was suppressed by miR-145-5p overexpression but promoted by the downregulation of miR-145-5p (Fig. 4B). The Transwell analysis suggested that the cell migration was also inhibited by miR-145-5p overexpression while promoted by the miR-145-5p downregulation (Fig. 4C and D; $\mathrm{P}<0.01$ ).

The regulated function of TAGLN2 in $B C$. To analyze the regulated function of TAGLN2 in BC, the TAGLN2 siRNA was transfected into the 5637 cells (Fig. 5A). Moreover, the MTT and Transwell assay were conducted to identify the proliferation and migration. The results suggested that the knockdown of TAGLN2 significantly inhibited the proliferation and migration of 5367 cells (Fig. 5B-D; P<0.05). It indicated that TAGLN2 might have the carcinogenic effect in $\mathrm{BC}$ to some extent.

\section{Discussion}

Many scholars have proposed that miRNAs act as oncogenes or tumor suppressors through regulating the relative target genes in various cancers (21), and that the change in miRNA expression was related to the pathogenesis, metastasis and progression of human cancer (22). Among them, miR-145 plays a suppressive role in tumor progression and is involved in tumorigenesis. Furthermore, miR-145 with anticancer effect was identified in ovarian carcinoma (23), colorectal carcinoma (24) and non-small cell lung cancer (25). The same conclusion for BC was obtained in this study. Furthermore, it has been reported that miR-145 participated in various physiological and pathological rhythms in BCs, including differentiation (26), apoptosis (27), and invasion (28). Although miR-145 taking part in pathogenesis of $\mathrm{BC}$ has been reported 
in many studies, its regulated mechanisms on cell proliferation and migration in $\mathrm{BC}$ continue to be confused.

This study revealed downregulation of miR-145 in BC tissues. Moreover, the inhibiting effect of miR-145-5p on cell proliferation and migration were found in BC. In brief, all the results indicated that miR-145-5p had inhibitory effects on the pathogenesis of BC. Therefore, it is worth exploring BC relationship between miR-145-5p and its target gene.

In this study, TAGLN2 was found to be a potential target gene of miR-145-5p in BC through bioinformatics analysis. As a member of the calponin family of actin-binding proteins, TAGLN2 is an oncogene. Many investigations have detected that TAGLN2 has modulated cell proliferation, differentiation, migration and apoptosis (29). Additionally, Yoshino et al proved that downregulating TAGLN2 inhibited cell proliferation, migration and invasion activity in BC (19). We also affirmed that miR-145-5p directly targeted TAGLN2 in the present study. Moreover, we found that miR-145-5p overexpression brought about downregulation of TAGLN2 in BC. Furthermore, Transwell analysis revealed that TAGLN2 overexpression promoted $\mathrm{BC}$ cell proliferation and migration which was in keeping with the previous studies.

Besides, we also identified the relationship between clinicopathological characteristics and miR-145-5p expression in BC. It was found that the expression of miR-145-5p was closely related to lymph node metastasis, differentiation and vessel invasion $(\mathrm{P}<0.01)$. Follow-up experiments will be conducted and the prognostic analysis for these patients will continue to be further analyzed.

In conclusion, the present study emphasized that miR-145-5p suppressed TAGLN2 expression and contributed to cell proliferation and migration in $\mathrm{BC}$. This novel miR-145-5p/TAGLN2 axis may provide new therapeutic implications for BC. Future research needs to make full use of the potential impact of miR-145-5p on cancer treatment.

\section{Acknowledgements}

Not applicable.

\section{Funding}

This research was funded by the project of 2017 Capital Medical University Basic Clinical Research Cooperation (17JL37) from Capital Medical University (Beijing, China).

\section{Availability of data and materials}

The datasets used and/or analyzed during the present study are available from the corresponding author on reasonable request.

\section{Authors' contributions}

HZ contributed significantly to data analysis and manuscript preparation. MJ performed the data analyses and wrote the manuscript. QL performed the data analyses. $\mathrm{ZH}$ helped perform the analysis with constructive discussions. YZ contributed in the organisation of the experimental data. SJ contributed to the conception of the study. All authors read and approved the final manuscript.

\section{Ethics approval and consent to participate}

The study was approved by the Ethics Committee of Beijing Ditan Hospital, Capital Medical University (Beijing, China). Signed informed consents were obtained from the patients or guardians.

\section{Patient consent for publication}

Not applicable.

\section{Competing interests}

The authors declare that they have no competing interests.

\section{References}

1. Harb-de la Rosa A, Acker M, Kumar RA and Manoharan M: Epigenetics application in the diagnosis and treatment of bladder cancer. Can J Urol 22: 7947-7951, 2015.

2. Bid HK: Words of wisdom. Re: Markers predicting response to Bacillus Calmette-Guérin immunotherapy in high-risk bladder cancer patients: a systematic review. Eur Urol 61: 846-847, 2012.

3. Ploeg M, Aben KKH and Kiemeney LA: The present and future burden of urinary bladder cancer in the world. World J Urol 27: 289-293, 2009.

4. Ye F, Wang L, Castillo-Martin M, McBride R, Galsky MD, Zhu J, Boffetta P, Zhang DY and Cordon-Cardo C: Biomarkers for bladder cancer management: Present and future. Am J Clin Exp Urol 2: 1-14, 2014.

5. Jiang QQ, Liu B and Yuan T: MicroRNA-16 inhibits bladder cancer proliferation by targeting Cyclin D1. Asian Pac J Cancer Prev 14: 4127-4130, 2013

6. Xu X, Li S, Lin Y, Chen H, Hu Z, Mao Y, Xu X, Wu J, Zhu Y, Zheng $\mathrm{X}$, et al: MicroRNA-124-3p inhibits cell migration and invasion in bladder cancer cells by targeting ROCK1. J Transl Med 11: 276, 2013.

7. Zhang M, Zhuang Q and Cui L: MiR-194 inhibits cell proliferation and invasion via repression of RAP2B in bladder cancer. Biomed Pharmacother 80: 268-275, 2016.

8. Ichimi T, Enokida H, Okuno Y, Kunimoto R, Chiyomaru T, Kawamoto K, Kawahara K, Toki K, Kawakami K, Nishiyama K, et al: Identification of novel microRNA targets based on microRNA signatures in bladder cancer. Int J Cancer 125: 345-352, 2009.

9. Shin SS, Park SS, Hwang B, Kim WT, Choi YH, Kim WJ and Moon SK: MicroRNA-106a suppresses proliferation, migration, and invasion of bladder cancer cells by modulating MAPK signaling, cell cycle regulators, and Ets-1-mediated MMP-2 expression. Oncol Rep 36: 2421-2429, 2016.

10. Guo J, Cao R, Yu X, Xiao Z and Chen Z: MicroRNA-223-3p inhibits human bladder cancer cell migration and invasion. Tumour Biol 39: 1010428317691678, 2017.

11. Yu H, Duan P, Zhu H and Rao D: miR-613 inhibits bladder cancer proliferation and migration through targeting SphK1. Am J Transl Res 9: 1213-1221, 2017.

12. Egawa H, Jingushi K, Hirono T, Ueda Y, Kitae K, Nakata W, Fujita K, Uemura M, Nonomura N and Tsujikawa K: The miR-130 family promotes cell migration and invasion in bladder cancer through FAK and Akt phosphorylation by regulating PTEN. Sci Rep 6: 20574, 2016.

13. Cheng Y, Zhang X, Li P, Yang C, Tang J, Deng X, Yang X, Tao J, Lu Q and Li P: MiR-200c promotes bladder cancer cell migration and invasion by directly targeting RECK. OncoTargets Ther 9: 5091-5099, 2016.

14. Feng C, Sun P, Hu J, Feng H, Li M, Liu G, Pan Y, Feng Y, Xu Y, Feng K, et al: miRNA-556-3p promotes human bladder cancer proliferation, migration and invasion by negatively regulating DAB2IP expression. Int J Oncol 50: 2101-2112, 2017.

15. Stanier P, Abu-Hayyeh S, Murdoch JN, Eddleston J and Copp AJ: Paralogous sm22alpha (Tagln) genes map to mouse chromosomes 1 and 9: Further evidence for a paralogous relationship. Genomics 51: 144-147, 1998. 
16. Nohata N, Sone Y, Hanazawa T, Fuse M, Kikkawa N, Yoshino H, Chiyomaru T, Kawakami K, Enokida H, Nakagawa M, et al: miR-1 as a tumor suppressive microRNA targeting TAGLN2 in head and neck squamous cell carcinoma. Oncotarget 2: 29-42, 2011.

17. Xiao G, Xia C, Yang J, Liu J, Du H, Kang X, Lin Y, Guan R, Yan $\mathrm{P}$ and Tang S: miR-133b regulates the expression of the Actin protein TAGLN2 during oocyte growth and maturation: A potential target for infertility therapy. PLoS One 9: e100751, 2014.

18. Du YY, Zhao LM, Chen L, Sang MX, Li J, Ma M and Liu JF: The tumor-suppressive function of miR-1 by targeting LASP1 and TAGLN2 in esophageal squamous cell carcinoma. J Gastroenterol Hepatol 31: 384-393, 2016.

19. Yoshino H, Chiyomaru T, Enokida H, Kawakami K, Tatarano S, Nishiyama K, Nohata N, Seki $N$ and Nakagawa M: The tumour-suppressive function of miR-1 and miR-133a targeting TAGLN2 in bladder cancer. Br J Cancer 104: 808-818, 2011.

20. Livak KJ and Schmittgen TD: Analysis of relative gene expression data using real-time quantitative PCR and the 2(-Delta Delta C(T)) method. Methods 25: 402-408, 2001.

21. Yoshino H, Seki N, Itesako T, Chiyomaru T, Nakagawa $M$ and Enokida $\mathrm{H}$ : Aberrant expression of microRNAs in bladder cancer. Nat Rev Urol 10: 396-404, 2013.

22. Nair VS, Maeda LS and Ioannidis JPA: Clinical outcome prediction by microRNAs in human cancer: A systematic review. J Natl Cancer Inst 104: 528-540, 2012.

23. Wang L, Wu X, Wang B, Wang Q and Han L: Mechanisms of miR-145 regulating invasion and metastasis of ovarian carcinoma. Am J Transl Res 9: 3443-3451, 2017.
24. Salem SM, Hamed AR and Mosaad RM: MTDH and MAP3K1 are direct targets of apoptosis-regulating miRNAs in colorectal carcinoma. Biomed Pharmacother 94: 767-773, 2017.

25. Chen GM, Zheng AJ, Cai J, Han P, Ji HB and Wang LL: microRNA-145-3p inhibits non-small cell lung cancer cell migration and invasion by targeting PDK1 via the mTOR signaling pathway. J Cell Biochem 119: 885-895, 2018.

26. Fujii T, Shimada K, Tatsumi Y, Hatakeyama K, Obayashi C, Fujimoto K and Konishi N: microRNA-145 promotes differentiation in human urothelial carcinoma through downregulation of syndecan-1. BMC Cancer 15: 818, 2015.

27. Blick C, Ramachandran A, McCormick R, Wigfield S, Cranston D, Catto J and Harris AL: Identification of a hypoxiaregulated miRNA signature in bladder cancer and a role for miR-145 in hypoxia-dependent apoptosis. Br J Cancer 113: 634-644, 2015 .

28. Kou B, Gao Y, Du C, Shi Q, Xu S, Wang CQ, Wang X, He D and Guo P: miR-145 inhibits invasion of bladder cancer cells by targeting PAK1. Urol Oncol 32: 846-854, 2014.

29. Dvorakova M, Nenutil R and Bouchal P: Transgelins, cytoskeletal proteins implicated in different aspects of cancer development. Expert Rev Proteomics 11: 149-165, 2014

(i)(3) This work is licensed under a Creative Commons Attribution-NonCommercial-NoDerivatives 4.0 International (CC BY-NC-ND 4.0) License. 